\title{
A partial inverse problem for quantum graphs with a loop
}

Shengyu Guan ${ }^{1}$ and Chuan Fu Yang ${ }^{1}$

${ }^{1}$ Nanjing University of Science and Technology

May 5, 2020

\begin{abstract}
We consider the Sturm-Liouville operator on quantum graphs with a loop with the standard matching conditions in the internal vertex and the jump conditions at the boundary vertex. Given the potential on the loop, we try to recover the potential on the boundary edge from the subspectrum. The uniqueness theorem and a constructive algorithm for the solution of this partial inverse problem are provided.
\end{abstract}

\section{Hosted file}

A partial inverse problem for quantum graphs with a loop.pdf available at https://authorea. com/users/292900/articles/420741-a-partial-inverse-problem-for-quantum-graphs-with-aloop

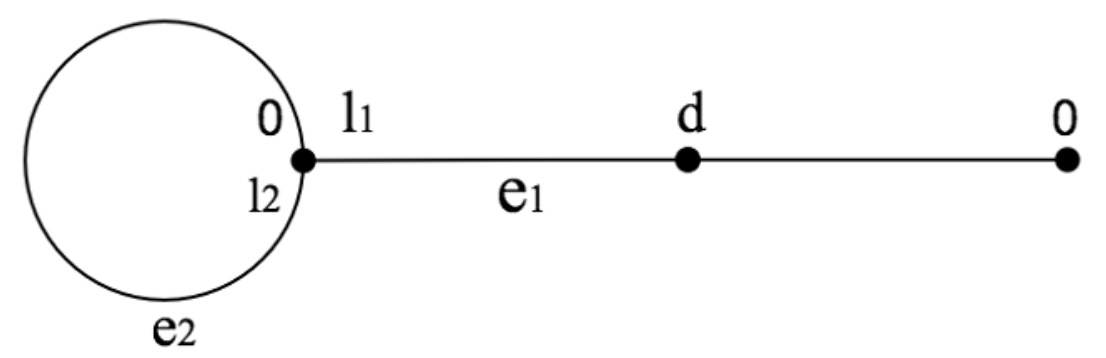


Fyt

444 


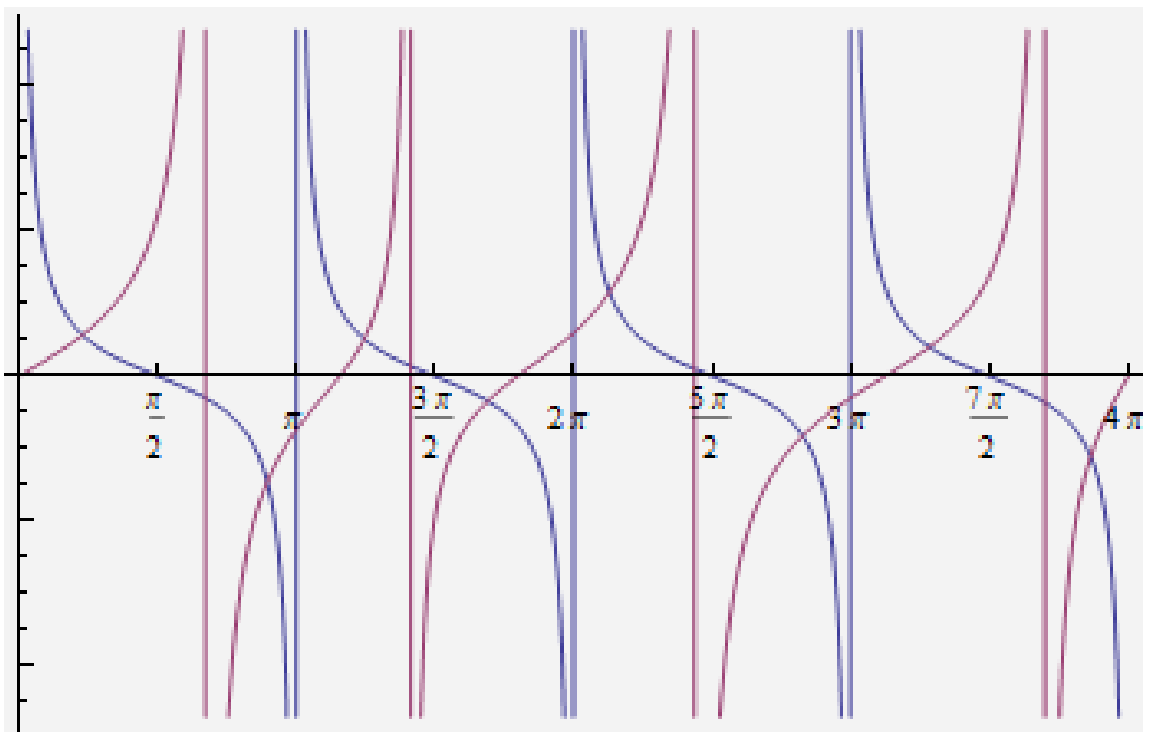

